\title{
Los caminos hacia una Sociología en Cuba. Avatares históricos, teóricos y profesionales
}

\section{TERESA MUÑOZ GUTIÉRREZ}

\section{Rescatando luces y sombras, no sólo un simple ejercicio intelectual}

Hasta el momento se han identificado algunas parcelas de la historia de la Sociología en Cuba, pero hay que escribirla y eso no se ha hecho. Sin embargo, constantemente nos asombramos de cuántas cosas estaban hechas en la década de los $60^{\prime}$ o lo que se produjo en los $70^{\prime}$ y las inquietudes resueltas en los $80^{\prime}$. Cuando se escriba esta historia hay que recordar los períodos de sombras, pero se van a rescatar muchas luces que también se han olvidado. La primera conclusión de este panel debe ser escribir la historia de la Sociología en Cuba, que hasta ahora no se ha hecho.(Juan Valdés Paz. Panel sobre el desarrollo de la Sociología en Cuba, celebrado en la Facultad de Filosofía e Historia de la Universidad de la Habana, el 9 de julio de 1999).

\section{El proceso de construcción de la ciencia: motivos y} limitaciones. Consideraciones preliminares

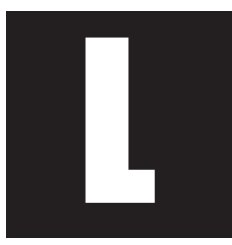

a historia de cualquier ciencia es importante, porque aporta una mejor comprensión de la naturaleza teórica de sus producciones y permite constatar su vínculo con lo más actual en el momento histórico preciso; estimula la aparición de nuevas reflexiones y porque trasmite el sentido de la continuidad de la indagación. En el caso de la Sociología en

\footnotetext{
* Dra. en Ciencias Filosóficas y Prof. Auxiliar del Departamento de Sociología de la Universidad de la Habana, Cuba.
} 
Cuba, estamos en presencia actualmente de una ciencia en un franco proceso de consolidación, pero que carece de una sistematización de su tradición histórica y que ha presentado una gran intermitencia institucional, factores que complejizan de manera significativa, el proceso de reconstrucción de su historia.

Mediante el rescate de la trayectoria que ha asumido la construcción del discurso sociológico en Cuba, salen a la luz nuevas perspectivas sobre el caudal de antecedentes intelectuales, revelándose contribuciones importantes en figuras y producciones teóricas antes ignoradas desde la perspectiva de esta ciencia, o solamente valoradas desde otras perspectivas.

Esta búsqueda metodológica y teórica, que se ha propuesto este saber, ha hecho que utilice sus propias armas reflexivas para autoevaluarse; utilizando la instrumentalidad que sugiere la Sociología del Conocimiento. Se hacen grandes esfuerzos por hacer una Sociología de la Sociología. Esta peculiar especialidad sociológica trata de explicar los cursos teóricos y metodológicos que experimenta este saber, a través de su relación con elementos tanto externos como internos a el. Así, ideología, economía, política y poder se presentan como determinantes de los cursos del conocimiento científico, al igual que las prácticas de su comunidad científica, los paradigmas compartidos, recompensas, criterios de validez, los problemas metodológicos, recursos científicos, formas de interacción, etc.

El logro de una reflexión de esta naturaleza dual obliga a distinguir lo sociológico en producciones que se han caracterizado por un espectro mucho más amplio de preocupaciones y por un carácter polidisciplinar; esto, sin abandonar la riqueza que le imprime a la Sociología del país, haberse mantenido constantemente en un intercambio con el espectro más amplio de las ciencias sociales.

La necesidad de reconstruir nuestra propia historia pasa por el camino de despejar muchas de las indefiniciones. Lo uno y lo otro deben marchar 
paralelamente, pero no podemos hacerlo desconociendo nuestra historia. Llevamos años intentándolo y hemos logrado avances. Claro que el logro de éstos retos está permeado por las tensiones evidentes que siempre han estado presentes, pero que actualmente, resultan aún más claras de resolver, el estatuto formal de la Sociología, su relación complicada y conflictiva con el poder y la necesidad real de hacer investigación social y producir pensamiento social sobre nuestra realidad actual y sus perspectivas.

Para insertarnos con criterio propio en el debate universal de las ciencias sociales sobre su lugar, futuro, aportes y su relación conocimiento - poder, se requiere de un posicionamiento gnoseológico que permita determinar los aportes que la ciencia ha realizado en el orden de la historia de las ideas, y en el de la historia de la ciencia. Es nuestra propuesta el tratar de hacer una Sociología de la Sociología en Cuba que se base en nuestra historia, pero que a la vez pueda enriquecer el arsenal de nuestra historia de las ideas incorporando la reflexión sociológica. A menudo, el sociólogo suele ser su propio historiador, pero no siempre puede serlo, en ocasiones se necesita del conocimiento de datos que sólo un historiador puede suministrar.

La historia de la Sociología en el país, ha estado marcada por muchos avatares, en algunos casos de signo traumático, donde se han conjugado razones institucionales, sociales, políticas, intelectuales, y todo ello, se ha traducido en momentos de retroceso, de saltos, de apertura y situaciones de cierre. Sin embargo, hoy la emergencia de las ciencias sociales se hace patente y necesaria. Las transformaciones introducidas por la nueva estrategia de desarrollo, condicionada por la crisis, han favorecido el desarrollo de las ciencias sociales y de la Sociología en particular, e incluso ejerce un efecto persuasivo en la dirección política de la Revolución sobre 
la necesidad de apoyar e impulsar el desarrollo de las ciencias sociales.

Ella ha logrado insertarse paulatinamente en el ámbito intelectual y cultural del país, con derivaciones y presencias en diversos terrenos: en el mercado, la cultura, la política, la opinión pública, y desde el punto de vista de la aludida institucionalización, contamos con el Departamento de Sociología de la Universidad de la Habana y de la Universidad de Oriente, en la Universidad de Las Villas, recién iniciaron un programa, que también se imparte en la Universalización ${ }^{1}$ en otras provincias del país. Existe y ha logrado importantes resultados en el terreno de la investigación el Centro de Investigaciones Psicológicas y Sociológicas (CIPS) dentro del Ministerio de Ciencias, Tecnología y Medio Ambiente (CITMA); pero además se cuenta con una carrera que renació y se fortalece, así como con una Maestría en Sociología y un Doctorado.

También la Sociología afronta dificultades que requieren en primer lugar de una comprensión por parte de los sociólogos y de todos los que en el país intervienen en los destinos de la ciencia; se trata de temas ausentes aún en la investigación y en la docencia; de dificultades en la introducción de resultados; de la no existencia de un debate sostenido al interior de la comunidad de sociólogos que permita la formación del consenso científico; de la falta de espacios de asociación que permitan fomentar la vinculación de las distintas instituciones que se dedican a este tipo de estudios; de la escasez de espacios de publicación; de la crisis de representatividad de la ciencia, manifiesta en la forma en que se utilizan los resultados de las investigaciones sociológicas y en las particularidades que presentan los vínculos existentes entre la ciencia y la política

\footnotetext{
1 Universalización de la Educación, proceso llevado adelante en el país, a partir del 2000 y por iniciativa del Comandante Fidel Castro Ruz. Mediante esta experiencia las universidades han creado sedes municipales en todas las provincias del país, donde se imparten un grupo de carreras. Es la forma de acercar la Universidad a la zona de residencia de los interesados y una oportunidad para que estas influyan de manera significativa en los desarrollos de los territorios. Beneficiarios de este programa son un grupo importante de jóvenes que por diversas razones no habían podido acceder a la enseñanza universitaria.
} 
(Hernández Morales y Díaz Mañalich,1995; Muñoz Gutiérrez, y Hernández Morales, 2001).

En el orden teórico y metodológico, esta ciencia en el país es deudora del desarrollo de la teoría a escala internacional, pues la Sociología en Cuba forma parte del sistema de ciencias, cuyo rango e historia son universales. Ello necesariamente, nos lleva a considerar más apropiado medir el desarrollo de nuestra disciplina con el nivel alcanzado internacionalmente. De cualquier modo, la madurez de la ciencia en Cuba es una madurez desigual en el conjunto de las ciencias sociales y dentro de la propia Sociología en las diferentes áreas de especialización.

Por muchos años luego de 1959, los estudios sociológicos permanecieron sumergidos al interior de estudios interdisciplinarios acerca de lo social, donde disciplinas científicas como la Historia, la Sicología o la Filosofía rectoraban los esfuerzos en este sentido. También en el campo de las Sociologías Especiales existen algunas con un camino recorrido más largo, como la Sociología Urbana, la Agraria, la del Trabajo, otras que se incorporan en la década del 80, como la de la Estructura Social o la de Género y el resto en los 90, Sociología de la Familia, de la Educación, la del Desarrollo, y todavía permanecen algunas sin una identidad propia o que todavía sus resultados son bastantes modestos.

Tampoco es posible, hablar de una Sociología en Cuba al margen de los derroteros que tuvo el movimiento teórico en el mundo, pero este proceso ha estado marcado por una especie de paradoja o contradicción, y es el hecho de que por una parte discurrió el desarrollo de esta ciencia y por otra parte, con gran independencia, transcurrió el desarrollo del marxismo y de la concepción materialista de la historia; es decir, de todo este aparato conceptual metodológico, que dentro de la tradición del marxismo, contribuía a la perspectiva del estudio de la sociedad en sus implicaciones y expresiones concretas. Esta antinomia marcó los derroteros 
de la investigación y la formación académica en el país (Muñoz, 2004).

Hay alrededor de cuatro aspectos que marcan la anterior interrelación en cuanto a la recepción de la teoría sociológica, en el contexto cubano. El primero nace de ese carácter polidisciplinar que en el terreno teórico ha presentado la construcción de la ciencia, lo que algunos estudiosos denominan como intrusismo teórico, entendido en el sentido de la transdisciplinariedad, pues dentro del pensamiento sociológico se reconocen derivaciones teóricas y preferencias temáticas de otras disciplinas afines, tales como el pensamiento filosófico, el económico, el político, el ético, etc.

El segundo elemento importante, es el eclecticismo teórico que ha marcado el desarrollo posterior, de forma tal que la Sociología no ha abrazado, de forma preferente, una sola de las diferentes corrientes que han coexistido dentro del pensamiento sociológico, sino que en ella confluye un pensamiento pluralista y enriquecedor, que en ningún caso ha sido algo negativo y de lo que en ocasiones no tienen conciencia los propios investigadores.

En el país no se han originado teorías que hayan aportado a la Sociología como cuerpo teórico, sino que se ha producido un proceso de recepción de los núcleos de pensamiento a nivel internacional. Dicha recepción manifiesta en la formación académica y en la labor investigativa, ha trabajado cada paradigma con la intencionalidad, en la mayoría de los casos de proveer al estudiante de una visión lo más amplia posible de los diferentes enfoques que sobre la realidad han coexistido y en la investigación de tratar de explicar la realidad social del país y otras realidades, imbricando los elementos de cada uno de dichos modelos recepcionados, por lo que se puede decir que este proceso no ha reproducido miméticamente ninguno de ellos. No obstante, es predominante la presencia del marxismo como enfoque teórico fundamental, en 
sus variantes, después de 1959 y sobre todo, después de la década del 90, se advierte una presencia del paradigma comprensivo (Peña Farías e Muñoz Gutiérrez, 2002).

El tercer rasgo es el peso relativamente pequeño, limitado y exiguo de los componentes teóricos dentro del desarrollo de la ciencia, pues si se compara con el desarrollo en el campo de lo metodológico, el primero ha sido muy pobre. El cuarto rasgo, es la dependencia y el solapamiento e intersección de nuestra Sociología con el campo del pensamiento crítico latinoamericano. Momentos de acercamiento e influencia como los protagonizados por el contrapunteo entre la Teoría Desarrollista y la de la Dependencia, y momentos de alejamiento total, como los que se producen en la segunda mitad del 70, y que tan graves consecuencias traen para la Sociología. El quinto aspecto a tomar en consideración, es el bloqueo de información. Es insuficiente el acceso a la bibliografía, tanto libros como resultados de investigación, y también existe una tendencia a la capitalización cuando se cuenta con ellos. ${ }^{2}$

Algunos especialistas coinciden en que, aún es inmaduro hablar de una Escuela de Sociología Cubana, no obstante esta haber alcanzado cierto grado de madurez en determinadas áreas de las Sociologías Especiales, y haber avanzado significativamente la formación de especialistas; razón por la que resulta estimulante comparar temporalmente el estado de su desarrollo en la década del 70 con los momentos actuales. Se plantea que no sería conveniente interesarnos por poner los apellidos de nacionales a nuestras sociologías, pues en ocasiones resultan más engañosos, que esclarecedores. "Uno de los inconvenientes, afirma un especialista, tiene que ver con un hecho simple, porque si asumimos como Sociología Cubana solo la que estudia a Cuba, estamos excluyendo los esfuerzos realizados por muchos sociólogos cubanos tratando de interpretar los procesos sociales y políticos de otros países", ${ }^{3}$ léase estudios sobre América Latina y los Esta2 Ver: Panel sobre el desarrollo de la Sociología en Cuba, 9 de julio de 1999, Departamento de Sociología, Facultad de Filosofía e Historia, Universidad de la Habana.

3 Ver: Palabras de Jorge Hernández, en Ob. Cit. 
Lo anterior, llevó a una interesante disquisición en el panel al que he hecho referencia, se usó el símil de que si quisiéramos medir la madurez científica de la Sociología con un vaso, resulta que para algunos está casi lleno, para otros casi vacío, y para los terceros, lo importante no es si el vaso está casi vacío o lleno, sino que lo que tiene dentro es una "emulsión". En este vaso hay más de un líquido y esos líquidos no todos combinan. ${ }^{4}$ Otra interrogante sería pertinente al respecto, qué pensamiento social está en la base de la construcción de la Sociología en Cuba, o de qué marxismo estamos hablando. Ambas cuestiones no han sido lo suficientemente estudiadas, y lo que se ha hecho no ha tenido suficiente divulgación, sin embargo en todos los casos, las tendencias presentes al interior de la Sociología, apoyan al proceso revolucionario y han demostrado su compromiso político con el Socialismo, independientemente de que entre ellas, puedan presentarse algunas divergencias teóricas o metodológicas.

Sin embargo, sería conveniente reflexionar acerca de si todos los que de una manera u otra nos relacionamos con la Sociología, tenemos conciencia de la naturaleza y el alcance de esta ciencia. Un maestro universitario cubano Roberto Agramante y Pichardo afirmaba en 1949, en el Congreso Internacional de Sociología celebrado en Oslo, que: "La Sociología ha de ejercer un decisivo influjo en el destino de nuestra nacionalidad, al dotar a ciudadanos, estudiosos y líderes de un órgano visual más perfecto para percibir la problemática en que inexorablemente se hallan inmersos" (Agramonte y Pichardo, 1949), la insistencia de este intelectual cubano, en la conveniencia de desarrollar esta ciencia es una constante desde un precursor como José Antonio Saco, o un fundador como Enrique José Varona o en figuras de talla tan elevada como el científico social Fernando Ortiz.

4 Ver: Palabras de Aurelio Alonso, en Ob. Cit. 
Muchos desde diversas perspectivas y variadas intenciones una vez más que otra, nos hemos referido a la historia de la Sociología en el país, pero pocos de manera sistemática hemos investigado rigurosamente este proceso. De ahí, que sobre la base de la investigación realizada en el Departamento de Sociología de la Universidad de la Habana, a partir del año 1997, por el Grupo de Estudios de Pensamiento Sociológico Cubano, es que me atrevo a exponer los criterios que se recogen en esta ponencia y que serán presentados en forma de libro el próximo año.

\section{Requisitos epistemológicos para la reconstrucción de la ciencia sociológica en Cuba}

Es posible reconocer ciertas dificultades epistemológicas para estudiar la Sociología en Cuba, que de una u otra manera, les he comentado con anterioridad en la ponencia, léase:

-La metodológica, que se fundamenta en la dimensión social polidisciplinar que presenta la ciencia, Para ello, se construyó un concepto de Sociología de matices académicos, que permite distinguir lo sociológico en producciones que se han enmarcado en un espectro mucho más amplio. Se entiende esta como el discurso que pretende explicar al actor social dentro de un contexto histórico, a partir de su interrelación con el hecho social e instituciones en su inserción en determinadas estructuras en funcionamiento, enfatizando en los factores sociales y en las consecuencias sociales de sus interacciones.

-La científica, expresada en la falta de sistematicidad en los estudios sobre este pensamiento, cuestión que nos lleva a ser cuidadosas en el terreno del establecimiento de propuestas científicas, pues el enriquecimiento paulatino del conocimiento acerca de la historia de la ciencia, puede implicar cambios en el cuadro general que tenemos de ella. 
-La histórica, manifiesta en la persistencia de una práctica científica institucional muy dependiente de la práctica social. Ello ha determinado que la realidad social haya propiciado o tronchado en determinados momentos el quehacer intelectual sociológico.

La diversidad de paradigmas teóricos y la búsqueda de una perfección metodológica constante han sido esfuerzos universales de la Sociología para lograr una identidad científica. Esto ha propiciado la maduración de la idea de una objetividad que no descanse en la neutralidad ideológicosocial, sino que se centre en reconocer la irrenunciable dimensión comprometedora que tiene esta ciencia en la sociedad y mantener una vigilancia epistemológica a través de una Sociología de la sociología.

No se puede olvidar que el sociólogo, en tanto intelectual, en tanto individuo, pertenece a un grupo que admite intereses, esquemas de pensamiento, problemáticas, en síntesis, todo un sistema de supuestos que está ligado a la clase intelectual, profesional, regional, como grupo de referencia. Quizás la presencia de estos elementos mediadores es lo que explica la intermitencia institucional que ha experimentado la Sociología en Cuba, de ahí que será necesario vincular toda su explicación a una realidad social que propicia o troncha en determinados momentos al quehacer intelectual sociológico.

No basta con identificar los representantes, instituciones y publicaciones cuya producción tiene carácter sociológico, o cercano a él, o revelar las preferencias teórico-metodológicas que se validan en el proceso de reflexión, en relación con las determinantes sociales del momento histórico y teniendo en cuenta las influencias del pensamiento a escala internacional y regional, ni de describir los discursos y sus portavoces.

Una historia del pensamiento sociológico en Cuba debe estudiar, además, el proceso de conformación e institucionalización de la Sociología cono disciplina científica, desde que cobra carácter autónomo a partir de 
la República Neocolonial hasta la actualidad; evaluando los antecedentes teóricos de este proceso, básicamente localizados en disciplinas a fines como la historia, la filosofía y la educación, donde ya se encuentra presente un modo de pensar sociológico asociado a la necesidad de buscar explicación a los problemas de la realidad cubana en términos de conductas en un marco social determinado, relación con instancias institucionales que se desarrollan en vínculos con las distintas esferas de lo social entendido en sentido amplio.

Para la Sociología, la comprensión de los referentes sociales e ideológicos de los discursos, sus portavoces y sus prácticas resulta imprescindible, sino ella misma estaría desideologizándose. El esfuerzo está dirigido al logro final de una autognosis de la Sociología en Cuba y para este fin resulta imprescindible analizar el proceso de conformación como disciplina científica, lo que necesariamente incluye el estudio de:

-La Conexión de la Sociología en Cuba con los cursos de la sociología a nivel internacional o regional (América Latina).

-La conformación a nivel internacional de un quehacer intelectual, sociológico o cercano a él, que reconozca, una tradición de pensamiento sociológico en Cuba; la posible búsqueda y defensa de un espacio independiente y específico teórico, metodológico y profesional para la Sociología y constate la existencia de polémicas respecto a paradigmas compartidos o en pugnas, entre los pensadores cubanos.

-La reconstrucción del proceso de su institucionalización, que recoge la existencia de marcos estables para la publicación sociológica, la inclusión de la Sociología como curriculum de las universidades en Cuba o en otros niveles de enseñanza, la producción de manuales para la enseñanza de esta disciplina y la celebración de eventos y 
-El establecimiento de criterios oficiales de cientificidad, de aceptabilidad y de reconocimiento de los aportes, es decir, las prácticas de una comunidad sociológica en Cuba.

Todo lo anterior posibilitará iniciar la superación de una ruptura que se ha establecido entre la tradición sociológica anterior al triunfo de la Revolución y el rescate que se ha intentado hacer de esta disciplina en el proceso revolucionario y evaluar los vínculos interdisciplinarios en los que ella se desarrolla.

\section{Esfuerzos de periodización}

Casi 10 años de trabajo docente e investigativo en el Departamento de Sociología de la Universidad de la Habana en esta temática, permiten identificar cuatro momentos en el estudio de esta ciencia en el país, y caracterizarlos sintéticamente.

-De finales del siglo XIX a inicios del XX. Primeros pasos en la conformación. -De 1900 a 1930. Institucionalización parcial. Intentos.

-De 1930 a 1959. Expansión y diversificación académica. Ascenso.

-De 1959 hasta la actualidad. Sociología en la Revolución. Zigzaguees. En esta etapa se establece su estudio por décadas, para una mejor comprensión de las complejidades del proceso y se reconoce desde la segunda mitad de la década del 80 hasta la actualidad determinados signos de maduración y ascenso en la consolidación de esta ciencia.

El pensamiento social cubano, desde sus primeros ejercicios y experiencias intelectuales, se acercó a su entorno con una mirada, podríamos decir "sociológica", aún cuando esta disciplina no había nacido oficialmente en países desarrollados de Europa, como Francia e Inglaterra. 
Si ello fue así, es porque aquellos investigadores se preocuparon más por los problemas concretos de la vida, las relaciones sociales y la instituciones; que por asuntos filosóficos abstractos, teóricos y sumamente generales. Quiere decir, que desde su nacimiento este pensamiento se constituye por derecho propio en antecedente del quehacer sociológico en Cuba, Estas características, son las que han llevado a los estudiosos a plantear que nuestra tradición intelectual, ha tenido la peculiaridad de elaborar un tipo de ciencia aplicada a las problemáticas concretas de la sociedad.

Si se tiene presente esta idea, se hace evidente que la labor de historiar la Sociología en Cuba, cuenta con un vasto campo de estudio. Sin dudas, son numerosas las personalidades e instituciones que pueden contarse entre los que de algún modo tributaron y tributan al desarrollo y difusión de este saber científico. En el podemos reconocer producciones teóricas cercanas a la Sociología, por la crítica que se realiza a las estructuras sociales existentes y sus propuestas de transformación, su vocación empírica y el uso de métodos cercanos a la Sociología. Se debe añadir, que se impone el reconocimiento de una tradición ensayística cubana que hace coincidir en él (ensayo), la imaginación y el análisis desgarrado de la cotidianidad (Ubieta, 1996, p. 7); y además, por la presencia en estas reflexiones acerca de la realidad cubana, de justificaciones generalmente fundamentadas en una razón científica positiva que se respiraba en la época y que mantenía su tensión con las ideas humanistas e independentistas que se desarrollaban.

No es posible entender, los desarrollos de la Sociología en Cuba, sin comprender la naturaleza de sus antecedente teóricos en el pensamiento de prestigiosas figuras que durante el período colonial elaboraron una rica producción intelectual. Tales pensadores reflejaron a menudo en sus obras, los problemas sociales que afectaban al país, como la existencia de la esclavitud, el mestizaje, la trata, el proceso de formación de la nacionalidad, fenómenos de desajuste social, etc. Algunos otros abordaron factores que, a nivel de pensamiento estaban obstruyendo el acceso al verdadero saber científico y libre de esquemas. 
Tales elementos pueden hallarse en la obra de José Agustín Caballero (1762-1835), Francisco de Arango y Parreño, Félix Varela (1787-1853), José de la Luz y Caballero (1800-1862) y en la del que es considerado por algunos, el primer sociólogo José Antonio Saco (1797-1879). A Saco se le reconoce toda una labor intelectual, que se destaca por su erudición y por su carácter polemista. En muchos de sus escritos está presente el enfoque sociológico. Entre ellos pueden mencionarse "Las Memorias de la vagancia en la Isla de Cuba" (1830), donde aparece un análisis crítico de las causas de este fenómeno social en Cuba y brinda una inteligente y veraz reconstrucción de las costumbres de la época, además realiza recomendaciones acerca de las posibles vías para solucionar algunos de los problemas.

Saco denuncia como posibles causas de la vagancia diversos males sociales como el juego, las deficiencias del sistema educativo, el déficit de ocupaciones lucrativas, analiza instituciones tales como la familia y el estado. Sus propuestas no solo se apoyan en la observación, sino que utiliza otros métodos cuantitativos, para explicar la situación. Empadronamientos, estadísticas, son algunos de los que propone.

En sus escritos contra la anexión de Cuba a los Estados Unidos, podemos encontrar algunos análisis sociológicos En ellos, argumenta que los rasgos típicos de la nacionalidad cubana, de nuestra cultura se perderían y quedarían aplastados en casos de producirse una anexión a los Estados Unidos de Norteamérica. También en su "Historia de la Esclavitud" podemos encontrar "un buen material sociológico de este fenómeno de subordinación y estratificación social" (Agramonte, Op. Cit,. p. 19), aún cuando su comprensión del problema del negro al interior de la nacionalidad cubana, no sea la más adecuada.

Especial atención recibe el pensamiento de José Martí, pues como teórico por excelencia de la realidad social. 
Sus análisis y previsiones, desarrollados en numerosas obras, acerca de las civilizaciones norteamericana, centroamericana y sudamericana son verdaderos documentos sociológicos acerca de las fuerzas, principios, intereses, composición, estructura y vida de estas sociedades, imprescindibles para la construcción metódica de una Sociología Americana (Agramonte, Op. Cit,. p. 19).

El carácter analítico, programático y universalista del pensamiento de Martí, reflejo de su gran preocupación por la situación y el futuro de la Isla; sus concepciones políticas que lo llevan a la comprensión de la verdadera naturaleza del imperialismo y a la necesidad de la revolución; su proyecto de nación, que al ponerse en práctica, propiciaría el logro del progreso sociocultural y económico cubano; su enfrentamiento a la corriente positivista, derivado de su profunda vocación humanista y espiritualista, lo sitúan como referencia obligada en el tratamiento de los antecedentes de un pensamiento sociológico en Cuba.

De una forma u otra los exponentes que acaban de mencionarse por solo citar algunos de los más importantes-, marcan la trayectoria del pensamiento social cubano desde posiciones liberal reformistas a revolucionarias, en el período de la Colonia. Ellos signan la transición de un pensamiento en el que dominaba la escolástica, hasta el comienzo de la crítica contra ella misma, por su carácter rígido y esquemático; promoviendo cada vez más los estudios científicos y el valor de la experiencia como fuente del conocimiento. Y aunque la tirantez entre la ciencia y la religión continúa viva; estas propuestas constituyen sin lugar a dudas, el desmoronamiento de los principios básicos de la escolástica más conservadora. ${ }^{5}$

5 Ver. Monal Isabel. Mitos del pensamiento latinoamericano en sus contrastes con Cuba, en Memorias del Taller de Pensamiento Cubano, Ed. CREART, 1995, pp.28-29; Salermo Judith. Otra vez Fernando Ortiz, ahora, tras el rescate de su imaginación sociológica. Departamento de sociología, UH, 2000, pp.27-28, Trabajo de Diploma. 
El retozo de este pensamiento con esta ciencia se fue haciendo cada vez más maduro, a medida, que se incorporaban más elementos sociales a los análisis, y se situó sobre la disfunción, que los historiadores cubanos reconocen se produce en la Isla, entre una plena aprehensión de la Ilustración como movimiento teórico y el retraso de nuestras estructuras sociales. No olvidar, que el arribo a la Modernidad, siempre es asociado a la aparición de la Sociología, sin embargo en la Isla, se afirma, no había una "plena modernidad" en el siglo XIX, e incluso hay referencias a la existencia de una "modernidad deformada", característica de los países latinoamericanos (Torres-Cuevas, Varela, 1995). ${ }^{6}$

Si bien las estructuras sociales no respondían a los criterios de la Modernidad, el desarrollo intelectual andaba por esos caminos. Esta disfunción propició que se conformara poco a poco un arsenal que inicialmente no cumplió los requisitos académicos para definirse como Sociología, pero en el ejercicio real de lo que se abordaba, si presentaba una cercanía a esta ciencia y que poco a poco comenzó a beber de las fuentes originarias para enriquecer y actualizar sus análisis. Este desfasaje entre la preparación académica y el deseo de hacer una práctica sociológica, sólo era el inicio de una discontinuidad que caracterizara todo el camino de la Sociología en el país.

A fines del siglo XIX, el positivismo logra ser precisamente una corriente dominante en aquellos que más se acercan a la Sociología. Debido a su relación con la tradición científica experimental existente en Cuba y relacionada con los ideales independentistas y de Modernidad de la Isla, las ideas del self goverment, relacionadas con la erradicación del colonialismo; la necesidad de controlar las fisuras del sistema, cuyo correlato teórico se encontraba en las concepciones de orden y progreso que este pensamiento 
propugnaba; la necesidad del impulso a la industria y a la ciencia, se convierten en atractivas para los intelectuales cubanos. El positivismo presumía de un conocimiento de la Modernidad, que era precisamente lo que algunos deseaban para la Isla.

Si se analizan las necesidades y las características de la sociedad cubana decimonónica, se puede concluir, que el Positivismo se presentó como un pensamiento social crítico y progresivo. La dependencia económica, la falta de libertades ciudadanas, los males sociales existentes, encontraban vías de erradicación en el discurso positivista, ya fuera en el orden y progreso comtiano, pero sobre todo, en la doctrina spenceriana. La necesidad de reforzar la educación y la cultura de los cubanos, así como la necesidad de una óptima industrialización, formaban parte del discurso cubano en concordancia con las reflexiones del positivismo (Guía, 2001).

El clima positivista existente propició que la Sociología pudiera instituirse en la Universidad. Según Norteamérica, la Sociología positivista es la ciencia social que significa modernidad al estudiar los hechos sociales sin retórica y con un espíritu científico positivo. La creación de un espacio para la Sociología en la Universidad significaba un síntoma de Modernidad. Por esa razón, algunos pensadores cubanos comenzaron a utilizar esa palabra en sus discursos de denuncia social. En este caso se encuentran los trabajos de Enrique José Varona "El bandolerismo" (1880) y "El imperialismo a la luz de la Sociología" (1906). Estas mismas premisas epistemológicas se mantuvieron a lo largo de toda la primera mitad del siglo XX y propiciaron un avance en la institucionalización de la Sociología. Habría que preguntarse si el rigor científico de las propuestas eran tan pretenciosos o eficiente como los espacios.

La primera etapa en el desarrollo de la Sociología en Cuba, entonces, está marcada por la presencia más delineada ya de una perspectiva socio- 
lógica. Esta tiene su presencia en discursos que: someten a estudio la realidad social, las problemáticas colectivas, para realizar evaluaciones y elaborar propuestas de transformación; estudian los fenómenos sociales como instituciones; buscan las determinantes socio-históricas de los fenómenos; analizan los fenómenos en su interrelación con otros fenómenos sociales; estudian fenómenos y procesos de alcance social; aquellos que en alguna medida impactan a la sociedad en sus grandes estructuras, e inciden en sus funciones; estudian los grupos sociales, su funcionamiento, su interna jerarquía de poderes, el conjunto de papeles que desempeña cada miembro ante los demás y la influencia del colectivo sobre la sociedad; usan métodos, que aunque no exclusivos de la Sociología, si pasan posteriormente a conformar la metodología utilizada por los sociólogos para la recopilación y análisis de datos; son usados algunos términos sociológicos tales como sociedad, sistema, clase social, ajuste social, integración social, orden, progreso, etc; reconocen de forma clara y consciente la necesidad y efectividad de la ciencia sociológica.

Enrique José Varona se convierte en la figura paradigmática de esta etapa. ${ }^{7}$ Este pensador elabora un tipo singular de Sociología, la interesada en el perfeccionamiento social y en el análisis y resolución de las problemáticas nacionales. Abre el camino al desarrollo de una Sociología académica con la institucionalización de dicha ciencia en la Universidad de la Habana, en 1900.

Varona estudia una gran cantidad de problemáticas vinculadas a la realidad cubana: históricas, educativas, sociales, políticas, literarias e incluso económicas. En la mayoría de ellas, su perspectiva teórico y

\footnotetext{
7 Nota: de 1900 a 1916 Varona desarrolló la cátedra de Lógica, Psicología, Etica y Sociología en la Universidad. En 1916 y hasta 1925 ocupó dicha cátedra Sergio Cuevas Zequeira, quién respetó las bases académicas que sentó Varona, Sin embargo, en 1926 ocupó la cátedra Roberto Agramonte y Pichardo, quien introduce un nuevo programa de estudio, extiende la enseñanza de la Sociología a varias escuelas de la Universidad, elabora manuales y realiza una importante labor en instituciones internacionales vinculadas con la ciencia. También se extiende la enseñanza de la Sociología a los preuniversitarios y se elaboran textos para estos fines.
} 
metodológica es propiamente sociológica, ya que en ellas se plantea la problemática sobre el estatuto de la ciencia, su objeto de estudio, la necesidad de la ciencia, los métodos de investigación. Por la importancia que le atribuye al hecho social y por los conceptos que emplea en sus elaboraciones teóricas: institución, orden, progreso, sociabilidad, sociedad, sociología, asociación, ente social, trasmisión hereditaria, entre otros. En estas obras aparecen criterios de racionalidad y ordenación de la sociedad, manifestándose como un pensamiento afirmativo y organizador. La Sociología se concibe como un instrumento de transformación de la sociedad por la vía pacífica y un estímulo para el desarrollo científico, educativo e industrial, además se reconoce la necesidad de su institucionalización como ciencia independiente (Muñoz y Benítez, 2000, p.6).

La etapa que se extiende del 30 al 59, que hemos denominado de expansión y diversificación, se caracteriza porque se produce un ascenso en el desarrollo de la ciencia, aunque no consideramos que este sea un tema en el que ya puedan decirse las últimas palabras, por presentar todavía enormes brechas y acontecimientos particulares que quedan por descubrir e interpretar; nos arriesgamos a exponer una premisa que se mantuvo guiando la mayor parte de las ideas planteadas a continuación. Se trata precisamente del contraste que se fue haciendo cada vez más evidente a lo largo de estas investigaciones, entre las prácticas sociológicas llevadas a cabo en los predios académicos (quehacer sociológico desarrollado exclusivamente en los marcos de la Universidad y otros centros docentes oficiales durante la República Neocolonial desde el momento de su institucionalización en nuestro país) y lo que en torno a la misma ciencia produjeron figuras e instituciones extraacadémicas de forma muchas veces simultánea. Precisamente entre ellas, cobra fuerza el desarrollo de las ideas marxistas, con antecedentes en Cuba desde fines del siglo XIX, y que en los primeros años de la República van a contar con un pensamiento tan creador como el de Julio Antonio Mella. 
Las investigaciones realizadas constataron que la Sociología Académica u oficial, tiene un desarrollo ascendente -impulsado por figuras notables como Enrique José Varona, Roberto Agramonte y Elías Entralgo- en cuanto a la extensión de la asignatura por las distintas Escuelas de la Universidad y otras enseñanzas, al número de estudiantes que la recibían y a la actualización paulatina de sus fuentes teóricas. ${ }^{8}$ El plan propuesto por Agramonte en 1926, constituyó un verdadero ascenso para la trayectoria institucional de la ciencia, desde el punto de vista teórico, pues actualiza las fuentes teóricas del pensamiento sociológico, pero su concepción básica de análisis continuó siendo el positivismo; en pocas ocasiones se emitieron juicios críticos, y no se generaron propuestas creativas, sino que por lo general se repitió pasivamente el contenido de las obras consultadas. Además no logró trascenderse el nivel de divulgación de la teoría.

La Sociología que se hace, está marcada por, las condiciones sociales en franco proceso de maduración que aportan datos y hechos significativos, que justifican los usos de ella, como ciencia del análisis de lo social, que puede producir una explicación pretendidamente científica, empírica, con afanes normativos y de reforma social, que evoca al positivismo, en algunos casos, y en otros los autores se sitúan en una perspectiva crítica de defensa de los intereses de las grandes masas.

Roberto Agramonte y Pichardo, es básicamente un maestro de Sociología, que con su acción contribuyó a que el conocimiento sociológico se estructurara, creando espacios para la enseñanza, la investigación, el debate, las publicaciones. Realizó cambios en los programas de esta especialidad, elaboró manuales que muestran un panorama de la teoría sociológica, que si bien no tienen mucho de creatividad, si se constituyeron

\footnotetext{
8 “En sentido general, la enseñanza de la sociología en la Universidad de la Habana recorrió, no obstante sus limitaciones, un camino en todo sentido ascendente y progresivo. La disciplina se fue extendiendo en todos estos años por el recinto universitario, y dejó de ser algo desconocido". Moreno Autie, Anilia. La Enseñanza de la Sociología en la Universidad de La Habana, Trabajo de Diploma. Departamento de Sociología, Universidad de La Habana, La Habana, 1989, p. 54-55.
} 
en un instrumento de divulgación del conocimiento sociológico en esos momentos y contribuyeron al fomento de una cultura sociológica en el país (Pérez, 1999).

En 1933 comenzó a impartirse la Sociología Cubana, explicada por Elías Entralgo y Vallina y la Sociología Pedagógica en 1936, por Martín Rodríguez Vivanco. Estas nuevas adiciones respondían básicamente al estado de crítica e inconformidad que prevalecía en nuestro país, tanto por parte de las grandes colectividades, como por parte de la clase intelectual, ${ }^{9}$ el espíritu revolucionario, nacionalista y de cuestionamiento, se vio reflejado en la academia. El profesor Entralgo y Vallina realizó una extraordinaria labor pedagógica y de formación de nuevas generaciones de intelectuales, sin embargo, no hace Sociología puramente, en su caso hay un uso funcional moderno de esta ciencia para explicar la realidad histórica del país, de ahí su importante vínculo con el modo de hacer de E.J. Varona. Al revisar sus conferencias originales que impartía en el curso de Sociología Cubana, no se aprecian polémicas sobre el objeto de estudio, sus conceptos o su metodología. Acepta las propuestas de Agramonte y Pichardo y comienza a hacer un estudio de lo cubano entendido como institución social. Hace una Sociología del cubano, de la cubanidad, pero sin mucho rigor de lo social para explicar lo social. Otros factores explicarán los comportamientos sociales, que el asume como regulares y siempre presentes. Las caracterís-

\footnotetext{
9 Desde fines de la década del 20, comienza en el país lo que estudiosos han denominado el "despertar de la conciencia nacional", se produce un crecimiento de las actividades populares e intelectuales y un aumento de la denuncia social a través de la crítica de los males socioeconómicos y el caos político que invadía la Isla. Son numerosos los hechos que ilustran ya desde 1923, el nuevo estado sociopolítico que se generaba. En estos momentos se produce un rico movimiento intelectual evidenciado a través de las producciones de historiadores, literatos, economistas, filósofos, maestros; de la creación de un gran número de instituciones culturales y espacios de publicación, etc. Todo ello contribuyó a promover la cultura nacional y demostró que la intelectualidad y el movimiento revolucionario en el país, estaba a la altura de los tiempos. Ver: Colectivo de Autores. Historia de Cuba. La Neocolonia .Organización y Crisis (1899-1940), Ed. Política, La Habana, T.III, 1998, p.198; Soto Lionel. La Revolución del 33, Ed. Pueblo y Educación, La Habana, 1985, T.I,p.22; Roa Raúl. La Revolución del 30 se fue a Bolina, Ed. Ciencias Sociales, La Habana, 1976, p.11-58; Le Riverend Julio. La República. Dependencia y Revolución, Ed. Revolucionaria, La Habana, 1966, p.76; Guadarrama Pablo y Miguel Rojas. El pensamiento filosófico en Cuba en el siglo XX: 1900-1960, Ed. Felix Varela, La Habana, 1998, p.69.
} 
ticas psicosociales del pueblo cubano y de quieneslo formaron, constituyen el centro del análisis de este pensador.

La Sociología académica se caracteriza, de forma general, por el énfasis marcado en las propuestas teóricas de los clásicos de la ciencia, en detrimento de creaciones verdaderamente autóctonas, y de la aplicación y divulgación de métodos y técnicas para acceder a la realidad social. Su enfoque básicamente abstracto y positivista, los mantiene un tanto alejados de las polémicas del entorno social. ${ }^{10}$ La contrapartida de este fenómeno se encuentra en creaciones que se producen, de forma autónoma y espontánea, -y que de alguna forma tributan al pensamiento sociológico en Cuba- en el marco de instituciones y centros de debate científico ajenos a la academia, por parte de prestigiosos intelectuales cubanos. ${ }^{11}$

A grandes rasgos puede decirse que los exponentes de la Sociología extra académica, se sitúan como herederos directos de nuestra más liberal tradición de pensamiento; lo que se traduce, sobre todo, en la utilización de la ciencia como instrumento de crítica y solución de la problemática nacional, en ser vivos exponentes del gran espíritu humanista y de justicia

\footnotetext{
10 Ver: Ibídem.

11 Con estas ideas se pretende distinguir las prácticas sociológicas o cercanas a la Sociología que se producen fuera de la Academia Cubana, respecto a su trayectoria permanente en el marco institucional. Se ha considerado que este movimiento representa una posición alternativa y, con mucho, bastante superior a los cursos más comunes de nuestra ciencia en las instituciones educativas, durante el período republicano. Dentro de este movimiento independiente, y espontáneo, puede situarse incluso a Enrique José Varona, por el hecho de que muchas de sus creaciones y aportes fundamentales a la disciplina, fueron realizados en lo que Benítez denominara, labor extracátedra, y no dentro de la Universidad, donde sus actividades fueron intermitentes, y menos exitosas. Aquí se ubicaría también, a Don Fernando Ortiz que ha sido el modelo básico utilizado para la definición de estas características; y quedaría para investigaciones futuras, el hecho de si pueden sumarse otras figuras como Raúl Roa, Carlos Rafael Rodríguez, Fidel Castro; entre otras personalidades que a través de la historia o el discurso político, observaron la realidad nacional y nuestras instituciones, con un enfoque crítico, y científico; a la par que propusieron alternativas para la solución de los males de nuestro país. Ver: Benítez Pérez, Elzbieta. Enrique José Varona y la génesis de la ciencia sociológica en Cuba, Trabajo de Diploma. Facultad de Filosofía e Historia, Universidad de La Habana, La Habana, 1999, p. 92; Salermo Izquierdo, Judith. Otra vez Fernando Ortiz: ahora tras el rescate de su imaginación sociológica. Trabajo de Diploma, U.H, 2000, p. 99-100; Muñoz, Teresa; Emilio Ichikawa; Aymara Hernández. Dos extremos metodológicos y una Historia de las ideas sociológicas en Cuba. Departamento de Sociología, Universidad de La Habana, 1996, p. 3-4. Inédito.
} 
social, y en mantener la postura militante que mantuvieron sus más cubanísimos antecesores, frente a los retos y conflictos sociopolíticos de la época; por eso llegan a constituir una Sociología aplicada al entorno social que se encuentra complementando las más diversas disciplinas sociales (Salermo Izquierdo, 2000, p. 99-100).

Desde el punto de vista teórico, pueden llegar a superar el positivismo, o bien asumiendo tendencias más progresistas dentro de la misma escuela de pensamiento, o asimilando otras teorías y concepciones del mundo. En su grado más radical pueden llegar a afiliarse parcial o totalmente al marxismo. Por lo general, se abstienen de la creación de sistemas teóricos y conocimientos abstractos, al estilo de otras escuelas sociológicas del mundo; aunque no desprecian la utilidad de la teoría.

Desde el punto de vista político pueden asumir desde un reformismo liberal burgués hasta las posturas más revolucionarias que implica la afiliación al pensamiento marxista. Marchan a la delantera de la Sociología oficial, y se sitúan en un nivel superior respecto a: las temáticas abordadas -actualizadas, más acordes con los fenómenos y conflictos de la época, ya sea a nivel nacional o internacional-; los métodos y técnicas para abordar la realidad social -variados y de distinta naturaleza; se exploran las vías posibles para captar la realidad nacional en su esencia misma-; la elaboración de propuestas nuevas, y la consiguiente contribución al saber universal con aportaciones autóctonas, muy relacionadas con las peculiaridades de nuestro contexto; y la utilización de la crítica como arma para la depuración de teorías y enfoques metodológicos.

Hacen uso de diversos espacios de debate -muchos de los cuales son creados por ellos mismos- en los que hacen públicas sus propuestas y evidencian el estado de opinión de la intelectualidad en torno a la problemática nacional. Hacen uso de las publicaciones en textos y revistas para la divulgación de sus ideas, y para la proposición de alternativas viables en 
pos de la solución nacional. Con todo, Ilegan a constituir un estímulo arrollador para las prácticas institucionales de la Sociología, hasta el punto de extender hacia ellas, poco a poco algunas de sus manifestaciones y propósitos. ${ }^{12}$

Es importante destacar que una de las estrategias de los catedráticos universitarios, fue la de nutrirse con lo mejor de nuestra intelectualidad, llamando a los salones e institutos del Magno Centro de Estudios, a los pilares básicos del saber científico social de la época, una de las figuras representativas y de talla universal, que tuvo un papel protagónico en los acontecimientos sociopolíticos y culturales del país fue Fernando Ortiz. En su pensamiento, la ciencia sociológica se ve profundamente enriquecida y va cobrando nuevos significados; así como un mayor alcance científico, en la relación que el sabio cubano establece con la Historia, la Antropología, la Etnografía...,teniendo como centro de sus reflexiones la cultura cubana, y todo lo relacionado con el concepto de transculturación. La perspectiva sociológica, es el lente a través del cual Ortiz contemplaba -en uno u otro momento, ya fuera como punto de partida, como fundamento, o como conclusión-, las más diversas cuestiones de su entorno social.

Sus aportes en este sentido, tienen que ver con la utilización de la ciencia como arma de la crítica, y el diagnóstico de la problemática nacional, y como vía de solución de los problemas. Hace alusión en la utilidad práctica de los conocimientos científicos sobre las relaciones humanas, y es por eso, y por su profundo rechazo a las teorizaciones, y a la elaboración de conocimientos abstractos, que su Sociología es básicamente aplicada. ${ }^{13}$

Se dice que la Sociología fue utilizada desde sus primeras prácticas como instrumento de comprensión y explicación, de diagnóstico y pronóstico, de legitimación y de cambio. Ella, mantuvo, además, un 
comprometimiento con la transformación social y la creatividad desde un análisis integrador de los procesos sociales. No es extraño encontrar, en su etapa inicial, dos rasgos fundamentales que regirán el curso del quehacer sociológico. Estos son precisamente, el intento por concebir de forma racional, la realidad fragmentada y convulsa del estadio moderno en el que se desenvuelve; así como el creciente empeño por obtener el prestigio y grado de cientificidad que ostentaban las ciencias naturales de la época (Muñoz; Ichikawa; Hernández, 1995, p. 3-4).

Se desarrolló también a fines de la década del 40 en Cuba, una práctica sociológica dirigida a realizar investigaciones basadas en la aplicación de la técnica del survey o encuesta. ${ }^{14}$ Esta técnica importada directamente del empirismo era un instrumento poco utilizado en el país (Gutiérrez Serrano, 1958), Esta técnica permitía analizar la opinión de las masas tanto comercial, como industrial, como políticamente; conocer sus gustos, simpatías y aficiones, todo esto, desde una perspectiva macrosocial. Esto tenía grandes ventajas, pues permitía conocer a través de una muestra seleccionada por el sistema de muestreo, los criterios de toda la población investigada, aún cuando esta no fuera homogénea. De ahí, que Raúl Gutiérrez afirmara que: "lo que es válido para la muestra lo es también para el total de la población". ${ }^{15}$

\footnotetext{
14 Raúl Gutiérrez Serrano, fue el más sistemático y conocido estudioso de la opinión pública en Cuba durante el período neocolonial, psicólogo de profesión y con una orientación "matemática" y "naturalista", graduado de la Universidad de Columbia en los Estados Unidos de Norteamérica, fue alumno de Paul Lazarsfeld. Vivió hasta 1987, plenamente identificado con el proceso revolucionario en el país, ocupó cargos en el Ministerio de Educación y posteriormente fue Profesor de la Universidad de la Habana, en la Escuela de Ciencias Políticas y luego en la Facultad de Filosofía e Historia. Ver: del Risco, Lidiet. La contribución del Departamento de Surveys al desarrollo de la Sociología en Cuba, Dpto de Sociología, UH, 2005. Tesis de Diploma en proceso de culminación. Es importante tener en cuenta que el uso de la encuesta y las técnicas cuantitativas en el país, es llevada adelante también por, Merle Davis en la década del 30, "La iglesia cubana en una economía azucarera"; Roury Nelson en 1947 "Rural Cuban"; La Asociación Católica Universitaria de Cuba, en 1956;, "Encuesta sobre religión de los cubanos", entre otros.
}

15 Ibidem, p. 17. 
Gutiérrez fue el fundador en 1946 del Instituto Cubano de Opinión Pública y de Sicología Aplicada, y en el 1947 de la Organización Técnica Publicitaria Latinoamericana. Los temas tratados evolucionaron hacia un mayor perfil social. En el primero se dedicó a la creación de una moderna organización encuestadora: un laboratorio de sicología donde se experimentaba con técnicas medidoras de actitudes y capacidades, se introducían conocimientos de sociología estadística, estudios demoscópicos y una sala de máquinas IBM, para el procesamiento de la información. Realizaron estudios acerca de: medición de capacidades con fines de contratación laboral, posteriormente fue ampliando su perfil con los temas de la productividad del trabajo, comunicación institucional imagen empresarial e impacto y penetración de la prensa hasta hacerse cargos de los Surveys de la Revista Bohemia en 1946; en esta fecha se incorpora la problemática electoral.

Indiscutiblemente aparece una nueva manera, no tradicional para las condiciones cubanas de hacer estudios sociales, los temas tratados se van haciendo mas sociológicos y de un carácter eminentemente empírico y con una vocación positivista, enfoque teórico que había predominado en toda la primera mitad del siglo XX, con raras excepciones. Por falta del arsenal teórico adecuado, Gutiérrez no trasciende de la constatación del hecho a su análisis causal (Romay, 2003, p.53-57). Su pensamiento evoluciona hacia una concepción marxista del mundo, pero mantiene su perfil cauntitativista en sus prácticas universitarias.

La cuarta etapa de desarrollo de la Sociología se ve impactada por el cambio radical que produce en todas las esferas del país la Revolución Cubana, el 1ero de enero de 1959. Las profundas transformaciones revolucionarias ocurridas en el país en los primeros años, y especialmente el impacto de la Reforma Agraria y la formulación de una nueva estrategia de desarrollo; plantearon la necesidad de realizar investigaciones sociales, 
que representaron un desafío para el Pensamiento Social Cubano. Sin embargo la Reforma Universitaria producida en 1962 (Martin Sabina, 2001, p. 3), no se hace cómplice de la necesidad de desarrollar la Sociología.

En un primer momento, porque las prioridades del desarrollo y el abandono del país de un sinnúmero de profesionales, exigieron otro énfasis y las universidades se lanzaron a formar ingenieros, médicos, maestros. En un segundo momento, porque la ciencia supone acumulación de conocimientos, consolidación de una masa crítica de especialistas y de una red de instituciones y publicaciones, así como la afirmación de una percepción del valor de la ciencia que contribuya a su fomento y promoción. Quiere esto decir, que no es suficiente con que existan necesidades sociales, problemas que el pensamiento debe asumir y descifrar, sino que también se requiere de una demanda social explícita que alimente la investigación social y la estimule. Visto de esta manera, la institucionalización de las Ciencias sociales y de la Sociología en particular tenía que recorrer un camino más largo y dilatado.

A lo anterior, se suma que la determinación del valor social de esta ciencia no siempre ha sido percibido con la misma claridad con que se ha apreciado en otras ciencias, lo que explica ciertas discontinuidades que se observan a lo largo de este proceso ${ }^{16}$ (carreras, instituciones, publicaciones que cerraron por períodos, la atribución de funciones limitadas a otras disciplinas, la sobre valoración de unas en detrimento de la otras), la intromisión ideológica en el destino de unas más que en el de otras y la visible carencia de demandas de algunas disciplinas que ha sido necesario afrontar.

Es indudable, que las revoluciones constituyen procesos que marcan la génesis misma de los discursos de la teoría social y especialmente de la

16 Ver: Nuñez Jover, Jorge y José Antonio Toledo. “Las ciencias sociales en el proceso de la construcción del Socialismo”, en Rev. Cuba Socialista, No. 45, p. 3-4. 
reflexión sociológica. Los cambios sociales asociados a ellas producen impactos de gran importancia en la formación y maduración del pensamiento, así como en los procesos de institucionalización y profesionalización de la ciencia. ${ }^{17}$

El reconocimiento que tanto han reclamado las ciencias sociales no es la única posición de demanda para el éxito de una revitalización. Es indispensable, la construcción de una verdadera cultura científica como parte de un proceso más amplio que englobe a la sociedad, cuestión que en ocasiones no es lo suficientemente comprendida por las personas que desde las instituciones oficiales impulsan o dificultan estos procesos. Pero para que la ciencia pueda concertar su potencial social garantizando un impacto cultural, se requiere de un contexto social globalmente innovador, la creatividad es un hecho cultural y la ciencia tiene desde su propia lógica compromisos que atender en ese proceso de creatividad necesaria, que en ocasiones se presenta como enfrentamiento de posiciones, o silenciamiento de unas posiciones en detrimento de las otras.

Estudiosos de las ciencias sociales después de 1959, coinciden en dividir el período por décadas para lograr un estudio más preciso de esta etapa histórica. Lo anterior, ha permitido elaborar muchas de las ideas que se exponen en este trabajo y que permiten caracterizarla en general como un momento de zigzaguees e indefiniciones en muchos campos del desarrollo de estas ciencias, a pesar de la voluntad política y los resultados de la Revolución Científica y Cultural llevada adelante en el país.

\section{Década de los 60}

17 Ver: Muñoz Gutiérrez, Teresa y Aymara Hernández Morales. "Sociología y Revolución. La continuidad de una reflexión teórica impostergable", en Selección de Lecturas de Introducción a la Sociología, Ed. Felix Varela, T.III, p. 433. 
La mayoría de los especialistas la reconocen como un momento de explosión de las ciencias sociales que se caracteriza por un espíritu creador y de renovación, sin embargo, se plantea la contradicción entre el interés de potenciar la investigación social; y la manera estrecha de valorar la importancia de la ciencia sociológica. El investigar problemas sociales se convirtió en una necesidad en estos años; se obtienen importantes resultados investigativos, hay una incorporación bastante creativa del marxismo, aunque persisten las diferentes formas de entenderlo dogmatismo-creatividad.

El intercambio entre los investigadores y el personal encargado de trazar políticas y tomar decisiones prácticas es directo y rápido; está presente un vínculo estrecho y fructífero entre los intentos de hacer Sociología en el país, con el campo del pensamiento latinoamericano. Sin embargo, es evidente la falta de reconocimiento de la necesidad de institucionalización de la Sociología, de la comprensión de la especificidad de su naturaleza como ciencia, e incluso prima una concepción estrecha del sociólogo que en muchos casos lastra la potencialidad de la disciplina y que nos recuerda al auxiliar de investigación, tendencia que tanto critica CH. W. Mills en su obra "La imaginación sociológica".

Es de destacar el hecho, de que la Reforma Universitaria, realizada en 1962 no contempla la apertura de espacios institucionales para la Sociología, sin embargo, se impulsan importantes investigaciones de carácter interdisciplinarios, se crea en la Universidad de Oriente la carrera en 1959, pero solo logra completar una promoción y es cerrada. Luego en 1968, ante el auge de las investigaciones sociales se abre en la Universidad de la Habana un Departamento de Sociología con perfil básicamente investigativo, pero que después logra graduar sociólogos hasta 1976.

\section{Década del 70}


La fertilidad intelectual que se suele asociar a la década del 60 , no se experimenta en la misma medida en los años del 71 al 79. Este período se enmarca en un proceso de reordenamiento del Socialismo en Cuba. De un modelo de experimentación más autóctono, se pasa a una aceptación del modelo soviético de socialismo en casi todos los órdenes, y las ciencias sociales no son una excepción y la Sociología resulta de las más afectadas. Acontecimientos de relevancia se producen, el Congreso de Educación y Cultura en 1971, el I Congreso del PCC en 1975 y el proceso de institucionalización. De manera mecánica se asumió un modelo de marxismo, donde la fetichización de las tres fuentes y tres partes integrantes del marxismo de V. I. Lenin, conllevaron a la separación entre materialismo dialéctico y materialismo histórico e incluso trajo consecuencias muy negativas para la ciencia sociológica, pues se consensuó la pertinencia de sustituirla por el materialismo histórico, acusándose a sus postulados clásicos de ser inapropiados, por su carácter burgués.

Esto provocó que el marxismo soviético se convirtiera en la única corriente teórica del marxismo legítima, y que desaparecieran el resto de los referentes, se silenciasen, o se filtrasen por el prisma de la academia soviética oficial. Sin embargo, se mantuvieron algunos núcleos de intelectuales que no perdieron el contacto con una literatura más variada, e incluso al interior de las carreras de Ciencias Sociales y Humanísticas se enseñaban las distintas corrientes del pensamiento universal, aunque bajo el prisma muchas veces a priori de la crítica como corrientes de pensamiento burgués. Por otra parte, es interesante constatar que hubo en ese período una acumulación de recursos humanos, de la que resultaron muchas de los especialistas, que en la segunda mitad de la década del 80 fueron protagonistas de las reformas producidas en los programas universitarios, lo que incluyó la posibilidad de la reapertura de la carrera de Sociología.

Como resultado, las Ciencias Sociales quedan sometidas a la rectoría de la Filosofía y a un excesivo tutelaje ideológico que limitó los espacios para el debate y la aceptación y/o explicación de lo diverso. En el período, 
predominan los estudios descriptivos centrados en demostrar hasta qué punto se implementaba en Cuba el modelo socialista aceptado, estos desde un enfoque excesivamente cuantitativo y empirista. En otros casos, esta labor de investigación dejó un débil impacto en la realidad, teniéndose un criterio reproductivo de ésta, lejos de la perspectiva de cambio en contraste con la década precedente (Espina, 1995). Existió también un escaso intercambio con Latinoamérica, producido fundamentalmente por la descontextualización que se produce del pensamiento respecto a su realidad, es decir, se profundizó la relación Europa del Este-Cuba y se descuidó la de Cuba con su región. Por esta situación se experimenta escasez de contactos culturales que por supuesto redujeron la comprensión de la identidad latinoamericana en el país dentro de estas ciencias e hicieron escasear los canales de intercambio.

Resulta contrastante y corrobora la no claridad existente aún en Cuba acerca de la necesidad de la Sociología como ciencia, el hecho de que por una parte, en esta década se interrumpe la enseñanza universitaria, con la consecuente fuga de los sociólogos hacia otras instituciones o su provisional reorientación en los marcos de la Universidad, y sin embargo, ya desde fines de los 60 ha sido creado el Grupo de Desarrollo de Comunidades, el Instituto Cubano de Investigaciones y Orientación de la Demanda Interna (ICIODI), en 1971, y el Grupo de Opinión del Pueblo, todos con una orientación de investigación hacia problemas centrales del desarrollo en el país, entrando en la agenda de trabajo de la Sociología problemáticas como: la rural, de mercado, de demanda, de opinión, modo de vida, entre otros, y que perseguían la búsqueda de un impacto social.

Básicamente la Sociología era utilizada para obtener un conocimiento empírico, y se ponía en práctica todo su arsenal instrumental para la recopilación de información y brindar soluciones a problemáticas concretas, vinculadas con las transformaciones en el campo y la ciudad, la satisfacción 
de las necesidades materiales y espirituales del pueblo, la producción, hábitos de consumo, gustos y preferencias, orientación de la moda, etc. Estos centros estaban constituidos por equipos interdisciplinarios y por lo regular al sociólogo se le reservaban funciones auxiliares, existiendo un escaso impulso a la teoría. En el caso del ICIODI, según afirma su director Eugenio Balari, cuando se refiere a que este organismo se formó en la práctica, combinando con mucha táctica, "la intelectualización de los problemas con los aspectos prácticos-operativos" ${ }^{18}$ Se ejecutan trabajos concretos acerca de la realidad cubana.

\section{Década del 80 (segunda mitad) hasta la actualidad}

La Sociología ha experimentado un paulatino proceso de fortalecimiento tanto en el terreno de la formación, como de la investigación. El año 1986 marca para Cuba el inicio del proceso de Rectificación de Errores y Tendencias Negativas, abortado en el 90 por la caída del campo socialista y el inicio del Período Especial, que provoca una profunda crisis en la sociedad cubana. Todos estos factores actúan de manera particular sobre los destinos de las Ciencias Sociales y de la Sociología en particular. Considero de lo más importante a destacar, el rescate paulatino y con contradicciones que se va produciendo de la naturaleza crítica de las ciencias sociales, de la problematización y el reconocimiento de las contradicciones sociales como hechos reales de la sociedad cubana.

Desde el punto de vista de la institucionalización, asistimos en la década del ochenta a los noventa a un proceso de avance, que va estar marcado por la reapertura del Departamento Docente de Sociología en la

18 Ver: Balari Eugenio. “Cinco años de trabajo del ICIODI", p.9-10. Material mimeografiado.

19 El Departamento de Sociología de la Universidad de la Habana se reabre en 1984., desde su desaparición en 1976, momento en que se crea el Ministerio de Educación Superior. Se produce una reestructuración de la enseñanza superior apareciendo la 
Universidad de la Habana, ${ }^{19}$ dirigido por el Lic. Euclides Catá Guilarte y posteriormente en la Universidad de Oriente, dirigido por Dr.Miguel Matute. Se crea el Centro de Investigaciones Sicológicas y Sociológicas en aquel momento de la Academia de Ciencias de Cuba, y hoy perteneciente al Ministerio de Ciencia Tecnología y Medio Ambiente, bajo la dirección de la Dra. Angela Casaña Mata, su antecedente en 1979 fue un departamento dentro del Instituto de Ciencias Sociales de la Academia de Ciencias. También se produce la creación a partir de 1995 de revistas, que aunque ninguna son estrictamente sociológicas, si abren un espacio a esta ciencia y donde los sociólogos publican con bastante frecuencia, Temas, Contracorriente y Debates Americanos, entre otras. Como afirmamos la crisis impacta de manera positiva a las ciencias sociales cubanas y la Sociología va a tener sus beneficios.

Significativa resulta la celebración en 1991 del XVIII Congreso de la Asociación Latinoamericana de Sociología (ALAS), bajo la coordinación del entonces director del Centro de Estudios de América, Dr. Raúl Suárez Salazar, espacio en primer lugar de encuentro de sociólogos cubanos y estudiantes de varias generaciones y de ellos con la comunidad académica internacional y principalmente latinoamericana, con la que se había producido un relativo aislamiento después de los setenta. Es de destacar, que este Congreso activa la comunidad de sociólogos y sociólogas cubanas, se presentan trabajos del Departamento de Sociología de la Universidad de la Habana y de Oriente; tanto de profesores como de estudiantes; del Centro de Investigaciones Psicológicas y Sociológicas, de Planificación Física, así como, de otros especialistas de las Ciencias Sociales en el país, que

\footnotetext{
Facultad de Filosofía e Historia, con las carreras mencionadas en su nombre, pero la Sociología entra en liquidación y desaparece como carrera universitaria. Al reabrirse el Departamento, se comienza a impartir la Sociología como una especialización de la carrera de Filosofía, algo bastante inusual, y no es hasta 1900, que comienza la Licenciatura en Sociología, la que ha transitado por varios planes de estudio y en la actualidad cuenta con 10 graduaciones. en la Universidad de la Habana y otras 10 en la Universidad de Oriente.
} 
tributan a la consolidación de los discursos de la Sociología en Cuba, y que desde importantes centros de investigación como el de Estudios de América, el de Estudios sobre los Estados Unidos, el de Antropología, el de la Juventud, estudian problemáticas cercanas a la Sociología o con un enfoque sociológico. Se hacen esfuerzos por la creación de la Asociación de Sociólogos de Cuba, en estos momentos pero no fructifican

La continuación de la década de los noventa trae consigo la consolidación de la formación de sociólogos y la aparición de los estudios de Maestría en Sociología por primera vez en el país, en 1997, en un proyecto conjunto de la Universidad de la Habana y la Universidad Autónoma de Barcelona, la que cuenta en la actualidad con dos ediciones. Asimismo en el año 2000 aparece el Doctorado en Sociología, pues con anterioridad, los sociólogos tenían que realizar maestrías y doctorados en el extranjero o sencillamente defender sus tesis en otras especialidades afines.

Hay un aumento del diálogo entre los investigadores y los hacedores de políticas, pero aún es insuficiente la introducción de resultados y no siempre resulta adecuada la comprensión y utilización que se hacen de éstos. En ocasiones se enfrentan transformaciones y cambios, sin que estos sean acompañados en alguna medida del criterio de expertos. Se mantiene la tensión entre la intención descriptiva de muchas investigaciones y los esfuerzos de otros por superar esquemas anteriores y proyectar una ciencia que no sólo sea capaz de producir análisis, sino elaborar pronósticos, proponer alternativas. Se revela en el orden teórico y metodológico una presencia del carácter multiparadigmático de la Sociología y las discusiones acerca de temas sensibles de la sociedad cubana aumentan, sin embargo, persisten prejuicios de carácter ideológico y políticos, muy vinculados a la concepción del marxismo que se sustenta, pues continúan enfrentadas 
diferentes maneras de asimilación del marxismo.

Cuando se analiza el estado de las ciencias sociales en Cuba, después de 1959, parece ya al fin existir un consenso que reconoce la existencia de momentos de crisis, aún cuando persisten posiciones que hacen lecturas demasiado triunfalistas de este proceso. Pienso que cualquiera de los dos extremos resultan poco productivos para los fines de una autognosis. De lo que se trata es de no perder la capacidad crítica que tienen las ciencias sociales y especialmente la Sociología y aprovecharla en función del perfeccionamiento de nuestra imagen de desarrollo de la ciencia y en el perfeccionamiento de la sociedad. No olvidar, que la realidad siempre es más rica que cualquier reflexión que podamos hacer sobre ella, y encasillarla es imposible. Tarde o temprano ella se nos revela en toda su potencialidad, sea esta negativa o positiva.

\section{Referencias}

AGRAMONTE Y PICHARDO, Roberto. Estado Actual de la Sociología en Cuba. 1949. (material fotocopiado)

ESPINA, Mayra. Tropiezos y oportunidades de la Sociología en Cuba. Rev. Temas, n.. 1, 1995.

GUÍA, Jessica. Viaje a la semilla: Positivismo y Sociología en la Cuba del XIX. Dpto de Sociología, UH, Tesis de Diploma, 2001.

GUTIÉRreZ SERRANO, Raúl. Seguiré utilizando el survey. Rev. Publicidad, órgano oficial del Colegio Nacional de Publicitarios, Año 1, n. 6, Sep-Oct. 1958.

HERNÁNDEZ MORALES, Aymara y DÍAZ MANALICH, María del Rosario. Sociología de la Sociología: un análisis crítico de esta ciencia en Cuba a partir de 1959. Dpto de Sociología, UH, Tesis de diploma, 1995.

MARTIN SABINA, Elvira. 40 Aniversario de la Reforma Universitaria. Rev. Cubana de Educación Superior, vol. XXI, n. 3, 2001. 
MUÑOZ GUTIÉRREZ, Teresa y BENÍTEZ, Elzbieta. Enrique José Varona y la génesis de la Sociología en Cuba. Ponencia presentada al Congreso de Historiadores Mejicanos y Cubanos, La Habana, 2000.

MUÑOZ GUTIÉRREZ, Teresa y HERNÁNDEZ MORALES, Aymara. Sociología y Revolución. La continuidad de una reflexión impostergable. In: Selección de Lecturas sobre Sociología y Trabajo Social, 2001.

MUÑOZ GUTIÉRREZ, Teresa. El legado de Carlos Marx. Una mirada desde la Sociología. Ponencia presentada en el Taller sobre los desarrollos del marxismo organizado por la Revista Cuba Socialista, 2004.

MUÑOZ GUTIÉRREZ, Teresa; ICHIKAWA, Emilio; HERNÁNDEZ, Aymara. Dos extremos metodológicos y una Historia de las ideas sociológicas en Cuba. Departamento de Sociología, Universidad de La Habana, 1995. Inédito.

PEÑA FARÍAS, Ángela; MUÑOZ GUTIÉRREZ, Teresa. Principales tendencias teórico metodológicas de la investigación sociológica en la década del 80 en Cuba. Ponencia presentada a la Conferencia de Filósofos y Científicos Sociales de Cuba y los Estados Unidos, junio 2002, Facultad de Filosofía e Historia, UH. Inédito.

PÉREZ, Milena. Roberto Agramonte y Pichardo: una de las vertientes en el desarrollo de la Sociología Académica en Cuba. Dpto de Sociología, UH, Tesis de Diploma, 1999.

ROMAY, Zuleika. La opinión pública en el ocaso de la Neocolonia Cubana. La Habana: Ed. Política, 2003.

SALERMO IZQUIERDO, Judith. Otra vez Fernando Ortiz: ahora tras el rescate de su imaginación sociológica. Dpto de Sociología, UH, Tesis de Diploma, 2000.

TORRES-CUEVAS, Eduardo. Félix Varela. Los orígenes de la ciencia y la conciencia cubanas. La Habana: Ed. Ciencias Sociales, 1995.

UBIETA Enrique. Ensayos de Identidad. La Habana: Ed. Letras Cubanas, 1996. 


\section{Resumo}

La ponencia realiza un panorama de la Historia de la Sociología en Cuba, a partir de la combinación de los enfoques de la Historia de las Ideas y de la Historia de la Ciencia. Brinda una caracterización de las etapas fundamentales por las que ha atravesado la ciencia sociológica en el país, combinando una mirada de lo universal, lo regional y lo nacional, destacando las especificidades de los procesos en Cuba, así como sus correlatos en Europa y en América Latina. Se atiende a los procesos de construcción de un pensamiento, de su institucionalización y de la formación de una comunidad científica.

Palabras-claves: Sociología en Cuba, perspectiva sociológica, preferencias teóricas y metodológicas, procesos de institucionalización, prácticas de su comunidad científica. 
The pathways towards a Sociology in Cuba: historical, theoretical and professional changes

\section{Teresa Muñoz Gutiérrez}

This article presents an overview of the History of Sociology in Cuba, combining approaches from History of Ideas and History of Science. It offers a characterization of the fundamental stages sociological science has undergone in Cuba, combining a look at the universal, the regional, and the local, underlining specificities of Cuban processes as well as their correlates in Europe and Latin America. It attends to the processes of construction of a type of thinking, its institutionalization, and the formation of a scientific community.

Key words: sociology in Cuba, sociological perspective, theoretical and methodological preferences, institutionalization processes, practices of the scientific community. 